\title{
Sex ratio and litter size in the guinea-pig
}

\author{
M. Peaker and E. Taylor \\ Hannah Research Institute, Ayr KA6 5HL, UK
}

\begin{abstract}
A significant relationship between sex ratio and litter size at birth was observed in the young of guinea-pigs caged singly from 8 weeks of age and during their first pregnancy; a male was present only at or around oestrus. Small litters ( $1-2$ young) had significantly more males than did large litters (4-5 young). Such a relationship was not apparent in animals housed communally, whether young or older or in their second pregnancy. Litters in late pregnancy (day 63) or at delivery were significantly smaller than at mid-pregnancy (day 35). A significant negative relationship was found between litter size and bodymass at birth and to at least 63 days of age. Growth rates during the period of lactation were lower in animals born in large litters. The possible adaptive significance of a plastic relationship between litter size and sex ratio is considered together with the physiological mechanism that might be involved.
\end{abstract}

\section{Introduction}

Evidence has been accumulating in a number of mammals that the sex ratio of offspring in utero or at birth may differ from one male:one female. For example, in gerbils (Meriones unguiculatus) and house mice (Mus musculus), it has recently been established that a mother's intra-uterine position, in relation to the sex of her neighbours, affects the sex ratio of her own offspring (Clark et al., 1993; Vandenbergh, 1993; Vandenbergh and Huggett, 1994). When apparent deviations from a sex ratio at birth of 1:1 have been detected, most attention has been directed towards interpreting any possible adaptive significance in the shift and of its compatibility with the hypothesis of Trivers and Willard (1973) on maternal condition, sex ratio and evolutionary fitness that parents should bias the sex ratio of their offspring towards the sex that will have the greatest effect on parental fitness. This approach, to considering any possible selective advantage of producing more of one sex than another in particular circumstances, has been critically reviewed (Clutton-Brock and Iason, 1986; Clutton-Brock, 1991) and the observed sex ratios have not been found to conform to the predictions of any single adaptive theory. Such approaches have also been made to relationships between sex ratio and litter size: might it be of selective advantage to produce fewer but bigger males or more, smaller females? We now report a relationship between litter size and sex ratio at birth in guinea-pigs (Cavia porcellus) which is affected by husbandry regimen, and discuss the physiological mechanisms that might be responsible for the phenomenon and for its plasticity.

The first indication that sex ratio may deviate from $1: 1$ was obtained in ten guinea-pigs that had been housed singly from puberty and examined daily for vaginal opening until midpregnancy; one of five males had been present but only during the period of vaginal opening. Six males and 27 females were born, with litter sizes ranging from two to five, although no record had been kept of the sex ratios of the individual litters.

Received 22 January 1996
Against the null hypothesis of the probability of each offspring being male was 0.5 and assuming that this probability was independent of the sex of its litter-mates, the sex ratio observed was significantly different $(P=0.0003)$. Therefore, data have been collected from animals being used in other studies and they are reported here.

\section{Materials and Methods}

Data were collected between 1987 and 1994 on litter sizes and sex ratios of guinea-pigs of the Dunkin-Hartley strain being used in other studies, housed singly in order to determine the time of mating, as in the group of animals described above, or housed in communal pens with other females and a male.

Individual data were collected for four groups: (i) animals caged singly, handled daily and examined for vaginal opening from 8 weeks of age until mid-pregnancy (a male present only during vaginal opening) (conception occurred at the first or second oestrus); (ii) animals housed communally, with other female guinea-pigs of the same age and a male, in floor pens ('behaviourally enriched' by the presence of plastic tubes and large piles of hay) from 8 weeks of age, handled only for routine pen cleaning and then caged singly in late pregnancy from the time of pubic relaxation; (iii) as (ii) but starting at 22 weeks of age; (iv) as (ii) but for a second pregnancy.

The mean bodymass of animals aged 8 weeks was $450 \mathrm{~g}$ and at conception, it was approximately $540 \mathrm{~g}$; while for those aged 22 weeks, it was $720 \mathrm{~g}$. All animals were kept under a photoperiod of $12 \mathrm{~h}$ light: $12 \mathrm{~h}$ dark and fed a 'breeder' diet, containing ascorbic acid, plus occasional green food. The sex of individual offspring was determined by visual examination within minutes or a few hours of birth, and checked at the time of weaning by another observer; the sex of any stillborn (that were not eaten by guinea-pigs) was also recorded.

Data were also collected on litter size in utero in guinea-pigs treated as (i) above and killed on day 35 (37 animals) or day 63 (47) of pregnancy. 
Another group of animals was studied in order to examine growth rate postpartum of animals born in litters of different size. Individuals were treated as in group (ii) above; mothers were kept in two groups of 13 and 14, with their young (41 males and 42 females in litters of $1-6$ ) that were marked at birth and then weighed at weekly intervals for nine weeks (the approximate time of puberty).

The data on sex ratio were analysed using a generalized linear model for binomial data with a logit link function (Collett, 1991).

\section{Results}

There was no apparent relationship between sex ratio and litter size in any of the groups housed communally (Table I). However, a relationship was apparent in the group of young animals in first pregnancy, housed and mated individually and handled daily until mid-pregnancy. The overall sex ratio was $48 \%$ males but small litters $(\leq 2)$ had significantly more males $(80 \%)$ than did large litters $(4-5 ; 41 \% ; P<0.05)$. Thus 12 litters of one or two offspring had 14 males and four females. In contrast, in 22 litters of four or five young, there were 38 males and 55 females (Table 1).

The size of the litters in 47 animals in late pregnancy (day 63) or at delivery (3.2 $\pm 0.16 \mathrm{SE}_{\text {; }}$ median 3) was significantly smaller than in 37 animals in mid-pregnancy (day 35) (3.8 \pm 0.16 ; median 4$)(P=0.01, t$ test). The frequency distribution (Fig. 1) shows the shift and the reduction in the proportion of litters of four to five young. It was calculated that approximately $30 \%$ of litters originally of four to five young were reduced in size between mid- and late pregnancy. An unequal distribution of fetuses between the two horns was found but without consistent bias to the left or right side. For example, in II animals bearing two to three fetuses, one uterine horn was empty in eight animals. Statistical analysis showed that abs (right-left) $\simeq 2 \pm 0.5 \quad(P<0.001$ in 22 animals). In five of the animals in mid-pregnancy (but none in late pregnancy), one to three intra-uterine masses, apparently conceptuses in a state of resorption, were seen; these were included in a comparison of litter size (above) (their exclusion had no material effect on the statistical significance).

Significant inverse relationships were apparent between bodymass at birth and litter size in both males and females $(P<0.05$ to $<0.001$ for the slopes of the regression lines in all groups); no differences were apparent between groups. The linear regression analyses for those animals in which growth postpartum was studied are shown (Table 2); singleton males were $27 \%$ (mean) heavier at birth than those born in litters of five. Growth rates up to 21 days of age were also significantly and negatively related to litter size. Therefore, the negative relationship at birth was even more marked at 7,14 and 21 days of age. For example, at day 21, males born in litters of one were $61 \%$ heavier than those born in litters of five. After this time, which corresponds to the period of lactation, there was no significant relationship between growth rate each week and litter size, although there was a tendency for the slope to become positive suggesting that those animals born in large litters were then growing at a greater rate than those born in small ones. However, the significant, negative relationship
Table 1. Sex ratio in litters of different size in groups of guinea-pigs kept under various physiological conditions and husbandry regimens

\begin{tabular}{lcccc}
\hline $\begin{array}{l}\text { Litter } \\
\text { size }\end{array}$ & $\begin{array}{c}\text { Number } \\
\text { of mothers }\end{array}$ & $\begin{array}{l}\text { Number } \\
\text { of males }\end{array}$ & $\begin{array}{c}\text { Number } \\
\text { of females }\end{array}$ & $\begin{array}{l}\text { Percentage of } \\
\text { males in litter }\end{array}$
\end{tabular}

Caged singly from 8 weeks of age; handled daily; male present at oestrus ${ }^{+}$; first pregnancy

$\begin{array}{lrrrr}\text { I } & 4 & 3 & 1 & 75 \\ 2 & 8 & 13 & 3 & 81 \\ 3 & 13 & 19 & 20 & 49 \\ 4 & 17 & 29 & 39 & 43 \\ 5 & 5 & 9 & 16 & 36 \\ \text { Total } & 47 & 73 & 79 & 48 \\ \text { Mean litter size } & 3.2 & & & \end{array}$

Housed communally from 8 weeks of age; male present throughout; first pregnancy

$\begin{array}{lrrrr}1 & 9 & 5 & 4 & 56 \\ 2 & 12 & 9 & 15 & 38 \\ 3 & 21 & 37 & 26 & 59 \\ 4 & 20 & 41 & 39 & 51 \\ 5 & 4 & 8 & 12 & 40 \\ 6 & 1 & 4 & 2 & 67 \\ \text { Total } & 67 & 104 & 98 & 51\end{array}$

Mean litter size $\quad 3.0$

Housed communally from 22 weeks of age; male present throughout; first pregnancy

$\begin{array}{lrrrr}1 & 3 & 1 & 2 & 33 \\ 2 & 4 & 4 & 4 & 50 \\ 3 & 10 & 14 & 16 & 47 \\ 4 & 4 & 8 & 8 & 50 \\ 5 & 2 & 3 & 7 & 30 \\ 6 & 1 & 3 & 3 & 50 \\ \text { Total } & 24 & 33 & 40 & 45\end{array}$

Mean litter size $\quad 3.0$

Housed communally from 8 weeks of age; male present throughout; second pregnancy

$\begin{array}{lrrrr}1 & 0 & 0 & 0 & \\ 2 & 3 & 3 & 3 & 50 \\ 3 & 5 & 8 & 7 & 53 \\ 4 & 7 & 12 & 16 & 43 \\ 5 & 8 & 18 & 22 & 45 \\ 6 & 2 & 5 & 7 & 42 \\ \text { Total } & 25 & 46 & 55 & 46\end{array}$

Mean litter size $\quad 4.0$

${ }^{\dagger}$ Examined daily for vaginal opening until mid-pregnancy; male present during opening; conception at first or second oestrus; 10 different males used.

between mass and litter size was maintained at all stages studied, up to and including day 63 of age. Thus, at the approximate time of puberty, males born in litters of five were $533 \mathrm{~g}$, whereas singleton males were, at $583 \mathrm{~g}$, nearly $10 \%$ heavier.

There were no significant differences in mass at birth or at any stage studied postpartum, between males and females born in litters of the same size (results not shown). Similarly, there 

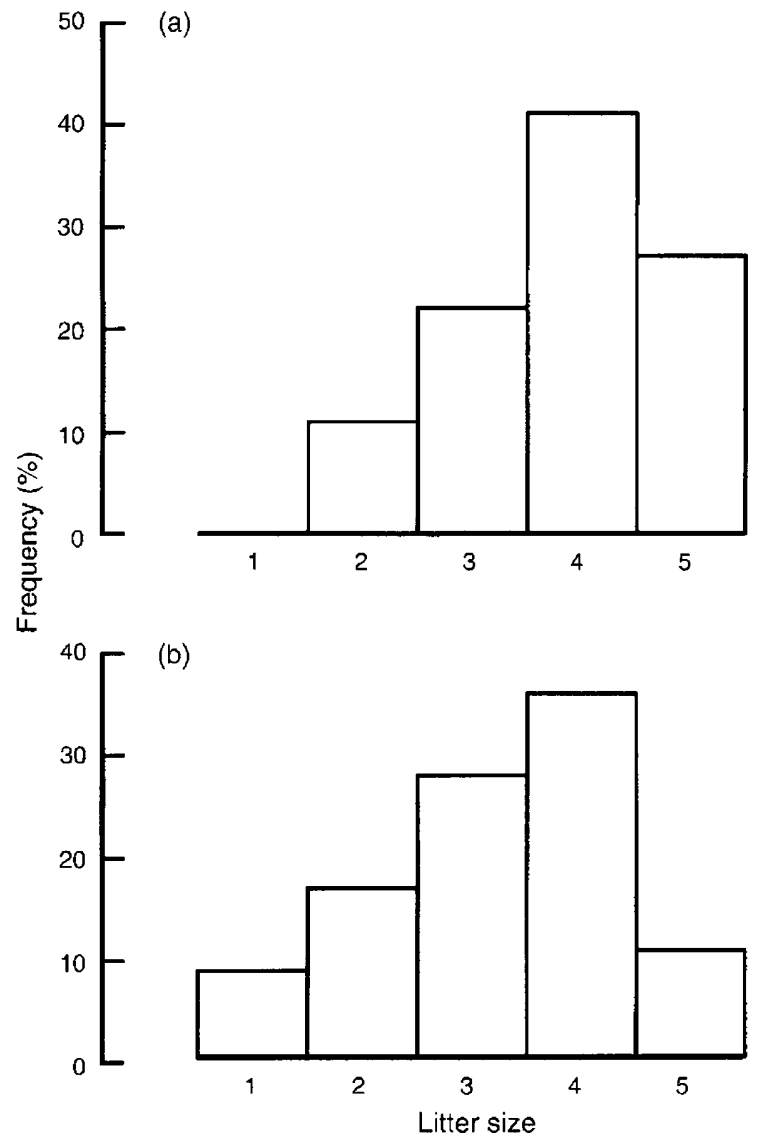

Fig. 1. Frequency distribution of litter size in (a) 37 guinea-pigs in mid-pregnancy (day 35) and in (b) 47 guinea-pigs in either late pregnancy (day 63) or at delivery.

were only small differences in the linear relationships between bodymass and litter size in males and females up to day 63 of age (Table 2).

In relation to the mother's parental expenditure, there was a significant, positive linear relation between the total mass of the young as a percentage of maternal mass immediately post partum $\left(y_{\mathrm{t}}\right)$ and litter size $(x)$ :

$$
y_{\mathrm{t}}=10.2+8.9 x(P<0.001)
$$

\section{Discussion}

There are a number of possible mechanisms by which sex ratio might be controlled in mammals, including differential sperm transport, events at conception, zygote transport, implantation, genomic imprinting and fetal resorption (Vandenbergh and Huggett, 1994; Krackow, 1995). The present observations of a relationship between sex ratio and litter size in guinea-pigs under one husbandry regimen but not others could be explained by control of fetal resorption. The evidence in favour of this view as the mechanism for controlling sex ratio in guinea-pigs is: the number of fetuses on day 35 of pregnancy exceeds the number on day 63 or at delivery; the number of corpora lutea exceeds the number of fetuses in late pregnancy (Eckstein et al., 1955); intra-uterine masses, apparently in a state of resorption, were seen in some animals on day 35 of pregnancy in animals treated in the same way as those in group (i).

Fetal resorption could be used to effect changes in sex ratio by two different physiological mechanisms: (i) determination of the sex of each conceptus within the uterus followed by sex-specific death and resorption (see Krackow, 1995); (ii) determination of the overall balance between the sexes in a litter with reduction of litters biased towards either males or females. The latter seems a more likely explanation to account for the excess of males in small litters and of females in large litters, since a proportion of males less than $50 \%$ in litters of four or five (in the group showing a high proportion of males in small litters) suggests a relative loss of large, predominantly male, litters. Therefore, an explanation for these findings, assuming a sex ratio at conception of $1: 1$, is that in male-biased litters originally of four to five young, there is reduction of litter size to one or two, leaving more small litters containing more males and the remaining large litters containing more females. Thus, there is no necessity to postulate sex-differential embryonic mortality at the level of the individual fetus to achieve a relationship between litter size and sex ratio, if, physiologically, the overall balance between the sexes can be detected. A number of mechanisms by which the mother might recognise the sex of her litter in utero have been suggested including production of testosterone by male embryos (see Krackow, 1995) although such discussions have been directed towards sex-specific death rather than the detection of biased litters and non-specific resorption. A physiological mechanism that detects the number of male (or female) embryos within the mother could form the basis of control of sex ratio in guinea-pigs. Such a mechanism could operate either within the whole animal or locally within a uterine horn.

It is interesting to note that in a group of animals with one transected uterine horn, studied by Eckstein et al. (1955), the raw data on the remaining horn show a similar trend to that reported here. Thus, 10 singletons were present. In four animals in which the number of ipsilateral corpora lutea was $>1$, the singletons were all males. In contrast, where there was a single corpus luteum, there were two males and four females.

Detection of intra-uterine sex ratio by the mother has been implicated in the reproductive strategy of another hystricomorph, the coypu (Myocastor coypus). In this species, Gosling (1986) found complete abortion of small litters in which female embryos predominated whereas large litters or small, male-biased litters were retained to term. This loss occurred about two-thirds the way through pregnancy and a similar timing, from about mid-pregnancy onwards, can be inferred in guinea-pigs.

The differences evident between the various groups in sex ratio in relation to the size of litter indicate a plasticity of physiological control. The proximate stimuli that might trigger processes leading to such a relationship are not known but could involve combinations of such factors as the stress of being housed singly, the stress of being handled daily, the absence of social visual signals arising from other guinea-pigs and age of the mother. It might be suggested that the greatest advantage of adjusting litter size might be expected in primigravid guinea-pigs mated soon after puberty while still growing. The chances of such mothers producing litters of 
Table 2. Linear regression analysis of bodymass and weekly growth rates in 41 male and 42 female guinea-pigs born in 27 litters of size 1-6

\begin{tabular}{|c|c|c|c|c|c|c|}
\hline \multirow[t]{2}{*}{$\begin{array}{l}\text { Age } \\
\text { (days) }\end{array}$} & \multicolumn{3}{|c|}{ Body mass $(\mathrm{g})$} & \multicolumn{3}{|c|}{ Weight gain (g) } \\
\hline & Intercept & Slope & $P$ & Intercept & Slope & $P$ \\
\hline \multirow[t]{2}{*}{0} & $\delta 125 \pm 6.2$ & $-6.4 \pm 1.48$ & $<0.0001$ & & & \\
\hline & $7115 \pm 8.3$ & $-4.4 \pm 2.30$ & ns & & & \\
\hline \multirow[t]{2}{*}{7} & o $184 \pm 7.6$ & $-13.3 \pm 1.81$ & $<0.0001$ & $59 \pm 5.6$ & $-6.9 \pm 1.35$ & $<0.0001$ \\
\hline & P $174 \pm 10.2$ & $-11.4 \pm 2.81$ & $<0.001$ & $58 \pm 6.1$ & $-7.0 \pm 1.69$ & $<0.001$ \\
\hline \multirow[t]{2}{*}{14} & of $279 \pm 11.9$ & $-21.2 \pm 2.85$ & $<0.0001$ & $96 \pm 6.8$ & $-8.0 \pm 1.63$ & $<0.0001$ \\
\hline & o $258 \pm 13.9$ & $-16.5 \pm 3.84$ & $<0.001$ & $84 \pm 6.6$ & $-5.1 \pm 1.82$ & $<0.01$ \\
\hline \multirow[t]{2}{*}{21} & o $365 \pm 14.6$ & $-25.7 \pm 3.50$ & $<0.0001$ & $87 \pm 6.3$ & $-4.9 \pm 1.51$ & $<0.001$ \\
\hline & † $338 \pm 18.2$ & $-20.3 \pm 5.01$ & $<0.001$ & $81 \pm 7.5$ & $-3.9 \pm 2.07$ & ns \\
\hline \multirow[t]{2}{*}{28} & $\delta^{\star} 402 \pm 15.8$ & $-23.4 \pm 3.78$ & $<0.0001$ & $35 \pm 7.4$ & $2.8 \pm 1.78$ & ns \\
\hline & $7373 \pm 19.3$ & $-17.8 \pm 5.33$ & $<0.0001$ & $48 \pm 9.9$ & $-0.8 \pm 2.72$ & ns \\
\hline \multirow[t]{2}{*}{35} & o $452 \pm 17.0$ & $-22.0 \pm 4.08$ & $<0.0001$ & $50 \pm 6.8$ & $1.3 \pm 1.64$ & ns \\
\hline & $q 409 \pm 20.5$ & $-13.9 \pm 5.67$ & $<0.05$ & $53 \pm 12.3$ & $-0.2 \pm 3.39$ & ns \\
\hline \multirow[t]{2}{*}{42} & o $494 \pm 19.4$ & $-20.5 \pm 4.65$ & $<0.0001$ & $40 \pm 7.0$ & $1.9 \pm 1.68$ & ns \\
\hline & $q 471 \pm 23.8$ & $-18.3 \pm 6.56$ & $<0.01$ & $62 \pm 8.8$ & $4.4 \pm 2.44$ & ns \\
\hline \multirow[t]{2}{*}{49} & o $535 \pm 24.0$ & $-19.5 \pm 5.76$ & $<0.01$ & $41 \pm 11.2$ & $0.7 \pm 2.69$ & ns \\
\hline & 7 $514 \pm 26.4$ & $-16.3 \pm 7.29$ & $<0.05$ & $43 \pm 9.7$ & $2.0 \pm 2.67$ & ns \\
\hline \multirow[t]{2}{*}{56} & d $564 \pm 23.8$ & $-15.7 \pm 5.71$ & $<0.01$ & $29 \pm 12.9$ & $4.0 \pm 3.09$ & ns \\
\hline & q $554 \pm 27.5$ & $-14.8 \pm 7.59$ & ns & $39 \pm 9.9$ & $1.5 \pm 2.72$ & ns \\
\hline \multirow[t]{2}{*}{63} & J $595 \pm 25.0$ & $-12.3 \pm 6.00$ & $<0.05$ & $31 \pm 7.4$ & $3.3 \pm 1.79$ & ns \\
\hline & 9 $595 \pm 29.7$ & $-13.7 \pm 8.19$ & ns & $42 \pm 12.4$ & $1.1 \pm 3.43$ & ns \\
\hline
\end{tabular}

The intercept (a) and the slope (b) (both \pm SE and with the $P$ value for the slope) of the regression equation, $y=a+b x$, where $y=$ bodymass $(\mathrm{g})$ or weight gain during the previous 7 days $(\mathrm{g})$ and $x=$ litter size are shown. $\mathrm{ns}=$ not significant $(P>0.05)$.

small individuals is clearly greater than in older animals of truly adult body size with metabolic reserves. Therefore, the postulated mechanism of litter reduction in large, male-biased litters might be more easily triggered in animals in which their anabolic effort during pregnancy is insufficient to produce large males in large litters.

There have been indications in other species of mechanisms by which maternal social conditions might lead to a reduction in litter size during pregnancy. For example, Pratt and Lisk (1991) showed that socially subordinate golden hamsters (Mesocricetus auratus) had smaller litters biased towards females. Also, possible hormonal mechanisms have been discussed for variations in the human sex ratio at birth (James, 1990).

Whatever the external triggers, and whatever the physiological mechanism, the result is that in young guinea-pigs, the overall level of parental expenditure is lower with a small litter while the mass of the young at birth and at the approximate time of puberty is increased. Whether such physiological plasticity is adaptive needs to be considered. Arguing teleologically, the guinea-pig is a polygynous species in which differential control of parental investment might be expected during pregnancy. For its bodymass, gestation is very long in this species, the young are precocious and parental expenditure during pregnancy is relatively great.

It appears that young female guinea-pigs under certain circumstances produce either a small litter, biased towards males, or a larger litter, biased, but not so markedly, towards females. The question then is: are males born in small litters at a selective advantage? The present results, as well as those of McKeown and MacMahon (1956) in C. porcellus and Rood
(1972) in the non-domesticated species $C$. aperea show that guinea-pigs in small litters are heavier at birth. The results also show that the growth rates of animals in large litters are lower during the relatively short lactation in this species. The young in large litters, that emerged from lactation lighter than those born in small litters, did not catch up until, at least the age of 63 days. Since dominant males have more reproductive success (Rood, 1972), a greater size at puberty and into adulthood may well be advantageous. However, there must clearly be a trade-off between size and number for selective advantage: small numbers of larger, intra-specifically competitive males versus larger numbers of smaller males more likely to provide a survivor of inter-specific predation.

These observations provide a number of testable hypotheses on the links between litter size and sex ratio that range from, and link, sociobiological factors to physiological control and its plasticity.

The authors are grateful to D. McNulty, D. Hirst and E. A. Hunter of the Scottish Biomathematics and Statistics Service and to S. Munro and A. C. McBlane for assistance. The Hannah Research Institute receives grant-in-aid from the Scottish Office Agriculture and Fisheries Department.

\section{References}

Clark MM, Karpiuk P and Galef BG (1993) Hormonally mediated inheritance of acquired characteristics in Mongolian gerbils Nature 364712

Clutton-Brock TH (1991) The Evolution of Parental Care. Princeton University Press, Princeton 
Clutton-Brock TH and Iason GR (1986) Sex ratio variation in mammals Quarterly Review of Biology 61 339-374

Collett D (1991) Modelling Binary Data. Chapman and Hall, London

Eckstein P, McKeown T and Record RG (1955) Variation in placental weight according to litter size in the guinea-pig Journal of Endocrinology 12 108-114

Gosling LM (1986) Selective abortion of entire litters in the coypu: adaptive control of offspring production in relation to quality and sex American Naturalist 127 772-896

James WH (1990) The hypothesized hormonal control of human sex ratio at birth - an update Journal of Theoretical Biology 143 555-564

Krackow S (1995) Potential mechanisms for sex ratio adjustment in mammals and birds Biological Reviews 70 225-241

McKeown T and MacMahon B (1956) The influence of litter size and litter order on length of gestation and early postnatal growth in the guinea-pig Journal of Endocrinology 13 195-200
Pratt NC and Lisk RD (1991) Role of progesterone in mediating stress-related litter size deficits in the golden hamster (Mesocricetus auratus) Journal of Reproduction and Fertility $92 \quad 139-146$

Rood JP (1972) Ecological and behavioural comparisons of three genera of Argentine cavies Animal Behaviour Monographs 5 1-83

Trivers RL and Willard DE (1973) Natural selection of parental ability to vary the sex ratio of offspring Science 179 90-92

Vandenbergh JG (1993) And brother begat nephew Nature 364 671-672

Vandenbergh JG and Huggett CL (1994) Mother's prior intrauterine position affects the sex ratio of her offspring in house mice Proceedings of the National Academy of Sciences USA $9111055-11059$ 